\title{
Coeficiente de asociación entre especies de selva mediana subperennifolia en Calakmul, México
}

\author{
Association coefficient between species of semi-evergreen forest \\ in Calakmul, Mexico
}

\author{
Alejandra Gómez Velázquez ${ }^{\text {a }}$, Alfredo Esteban Tadeo Noble ${ }^{\mathrm{b} *}$, Leonardo Beltrán-Rodríguez ${ }^{\mathrm{c}}$, \\ Enrique Guízar Nolazco a, Maribel Apodaca Martínez b, Ángel Leyva Ovalle a \\ ${ }^{a}$ Universidad Autónoma Chapingo, División de Ciencias Forestales, Texcoco, México. \\ * Autor de correspondencia: ${ }^{\mathrm{b}}$ Instituto Tecnológico Superior de Venustiano Carranza, Puebla, México, \\ Av. Tecnológico S/N, Col. El Huasteco, Lázaro Cárdenas, tel.: 746-843-57-53, alfredo.tadeo@itsvc.edu.mx \\ c Universidad Nacional Autónoma de México, Instituto de Biología, Jardín Botánico, \\ Laboratorio de Etnobotánica Ecológica, Ciudad de México, México.
}

\begin{abstract}
SUMMARY
Association coefficients measure interrelation level between the attributes of two species within populations. The objective of this work was to analyze the association patterns among the 10 species with the highest importance value index (IVI) in a semi-evergreen forest of Calakmul, Mexico. The 10 taxa were expected to be independently distributed within the community, therefore no interspecific association would occur. Systematically, 479 sampling units were established on a 500-ha surface in the ejido Gustavo Díaz Ordaz, Calakmul. Eighty-five tree species, 36 genera and 32 families were recorded. IVI was estimated, and with the 10 most important species, an $\chi^{2}$ analysis was performed to test independence in 45 possible combinations of species pairs. Cole's interspecific association coefficient was considered based on the $\chi^{2}$ significance test. The 10 most important species represent $47.98 \%$ of the IVI. The Cole association coefficient identified that 17 pairs, out of 45, were distributed in a dependent way regarding another species, while the rest were indifferent to the presence of these taxa. Bursera simaruba, the species with the highest IVI, was related to three species, while Metopium brownei (third position of IVI) was related to six taxa. It is proposed to apply this procedure as an independent though complementary statistical test to Ripley's $\mathrm{K}_{12}(\mathrm{t})$, to establish numerical criteria for the selection and analysis of spatial data.
\end{abstract}

Key words: Cole coefficient, spatial ecology, importance value index, Ripley's $\mathrm{K}_{12}(t)$, Synecology.

\section{RESUMEN}

Los coeficientes de asociación miden el nivel de interrelación entre los atributos de dos especies dentro de las poblaciones. El objetivo de este trabajo fue analizar los patrones de asociación entre las 10 especies con mayor índice de valor de importancia (IVI) en una selva mediana subperennifolia de Calakmul, México. Se esperaba que los 10 taxa se distribuyan de forma independiente dentro de la comunidad, por lo que no se presentaría ningún tipo de asociación interespecífica. Se establecieron 479 unidades de muestreo de forma sistemática en una superficie de 500 ha en el Ejido Gustavo Díaz Ordaz, Calakmul. Se registraron 85 especies arbóreas, 36 géneros y 32 familias Se estimó el IVI, y con las 10 especies más importantes se realizó un análisis de $\chi^{2}$ para probar la independencia en 45 combinaciones posibles de pares de especies; se utilizó el coeficiente de asociación interespecífica de Cole basado en la prueba de significancia de $\chi^{2}$. Las 10 especies más importantes representaron 47,98 \% del IVI. El coeficiente de asociación de Cole indicó que 17 pares de 45 se distribuyeron de forma dependiente a otra especie, mientras que el resto fue indiferente a la presencia de estos taxa. Bursera simaruba, especie con mayor IVI, se relacionó con tres especies, mientras que M. brownei (tercera posición de IVI) se relacionó con seis taxa. Se propone aplicar este procedimiento como una prueba estadística independiente pero complementaria a la $\mathrm{K}_{12}(t)$ de Ripley, a fin de establecer criterios numéricos para la selección y análisis de datos espaciales.

Palabras clave: coeficiente de Cole, ecología espacial, índice de valor de importancia, $\mathrm{K}_{12}(t)$ de Ripley, sinecología. 


\section{INTRODUCCIÓN}

La asociación interespecífica es una característica numérica de la formación, evolución y estabilidad de las comunidades vegetales (Janson y Vegelius 1981, Chai et al. 2016). También representa la reacción de dirección al interior de la comunidad entre las especies, en donde las ocurrencias mutuas expresan interacciones positivas (atracción) o negativas (repulsión) con miembros de otra especie que suceden en espacios determinados y que se pueden determinar mediante métodos de muestreo (Bray 1956, Hurlbert 1969).

Diversos métodos objetivos y cuantitativos se han desarrollado para determinar el grado de ocurrencia mutua entre especies, basados en el reconocimiento de tres niveles espaciales de relaciones bióticas: geográfico, comunal e interespecífico (Bray 1956). Los coeficientes de asociación son instrumentos importantes en el análisis de datos de diferentes aspectos ambientales relacionados a la interacción espacial entre organismos (Whittaker 1975, Su et al. 2015, Liu et al. 2017). Las medidas de semejanza tratan de evaluar el grado de interacción entre dos especies en una misma localidad bajo criterios de coexistencia (Hurlbert 1969, Janson y Vegelius 1981). La medida de asociación basada en datos presencia y ausencia de especies sacrifica información asociada a la abundancia, debido a que generalmente se analizan atributos ecológicos en forma independiente, como lo son la cobertura, densidad, biomasa o talla (Hurlbert 1969).

La prueba de Chi-cuadrada $\left(\chi^{2}\right)$ se ha utilizado como una medida de asociación interespecífica, ya que considera la dependencia de las frecuencias entre individuos que se comparan dentro de la muestra de una misma población (Hurlbert 1969). La hipótesis nula de distribución independiente de datos de especies se comprueba con una prueba de Chi-cuadrada de una tabla de contingencia $2 \times 2$, y los índices de asociación muestran la fuerza de las hipótesis alternativas acerca de que las especies coexisten más o menos frecuentemente de lo esperado (Cole 1949, MacKenzie et al. 2017).

La comprensión de las relaciones de asociación interespecífica entre especies arbóreas podría ayudar a revelar mecanismos de reemplazo en el proceso de desarrollo de las comunidades vegetales, base fundamental de la gestión forestal (Jiménez-Pérez et al. 2009, Chai et al. 2016); además de que promueve información sobre modelos de recuperación de vegetación (Jiménez-Pérez et al. 2009, Huang et al. 2017, Liu et al. 2017). La medición de asociaciones interespecíficas aporta a su vez ideas sobre interacciones y relaciones ecológicas entre especies y la dinámica de las poblaciones (Cole 1949, Liu et al. 2017). Los coeficientes de asociación interespecífica de especies arbóreas (como los índices de Jaccard, Sorensen y Ochai) han sido empleados en estudios de vegetación desde inicios del siglo XX para describir la semejanza entre comunidades y la asociación entre especies dentro de una comunidad (Shaukat et al. 2014, Chai et al. 2016, Liu et al. 2017), y aun cuando el coeficiente de Cole es un método espacial pionero, sencillo y estadísticamente robusto, se ha utilizado en un número considerable de investigaciones, pero la mayoría de ellas se han desarrollado en el continente asiático y en bosques templados (Chai et al. 2016, Huang et al. 2017).

El objetivo del presente estudio consiste en analizar la asociación entre las principales especies arbóreas que componen la selva mediana subperennifolia en el ejido Gustavo Díaz Ordaz, México, bajo el supuesto de que los diez taxa más importantes descritos de acuerdo con el índice de valor de importancia se distribuyen de forma independiente dentro de la comunidad, por lo que no presentan ningún tipo de asociación interespecífica.

\section{MÉTODOS}

Área de estudio. El ejido Gustavo Díaz Ordaz se ubica en el municipio de Calakmul, en el estado de Campeche, México, desde $89^{\circ} 09^{\prime} 30^{\prime \prime}$ a $89^{\circ} 13^{\prime} 08^{\prime \prime}$ longitud Oeste y desde $18^{\circ} 26^{\prime} 10^{\prime \prime}$ a $18^{\circ} 27^{\prime} 10^{\prime}$ " latitud Norte. El clima en la zona es cálido subhúmedo con lluvias en verano, con temperatura media anual que oscila entre de los 24 y $26^{\circ} \mathrm{C}$ (INEGI 2017) y una precipitación media anual de 1.076 mm (Martínez y Leal 2002). El sustrato es de tipo sedimentario, en tanto que los suelos predominantes en esta área corresponden a leptosoles y gleysoles (INEGI 2017). La vegetación pertenece a selva mediana subperennifolia (Miranda y Hernández-Xolocotzi 2014) con presencia frecuente de Bursera simaruba (L.) Sarg., Caesalpinia gaumeri Greenm., Lisiloma latisiliquum (L.) Benth., Piscidia piscipula (L.) Sarg., Coccoloba cozumelensis Hemsl., Guettarda combsii Urb., Simarouba glauca DC. y Metopium brownei Urb. (Rzedowski 2006).

Procedimiento de muestreo. Se realizó un muestreo distribuido de forma sistemática en una superficie de 500 ha, donde se establecieron 479 sitios de $250 \mathrm{~m}^{2}$ de forma rectangular de $10 \times 25 \mathrm{~m}$, separados entre los centros de cada sitio a $50 \mathrm{~m}$ de sur a norte y $200 \mathrm{~m}$ de este a oeste (figura 1), con un área de muestreo de 11,975 ha. La medición de todos los individuos con diámetro normal (Dn) mayor o igual a $5 \mathrm{~cm}$ se midió con una cinta diamétrica. Los ejemplares fueron procesados y herborizados (Lot y Chiang 1986), para su posterior identificación taxonómica y resguardo en el Herbario Hortorio "CHAPA" del Colegio de Postgraduados Campus Montecillo. La nomenclatura botánica se homogenizó mediante la base de The International Plant Names Index (IPNI).

Importancia estructural. Se utilizó el índice de valor de importancia relativo (IVIr) para jerarquizar la dominancia estructural de los elementos arbóreos en la selva bajo estudio. El cálculo del IVIr se determinó mediante la ecuación 1 (Liu et al. 2017), expresado en porcentaje. 

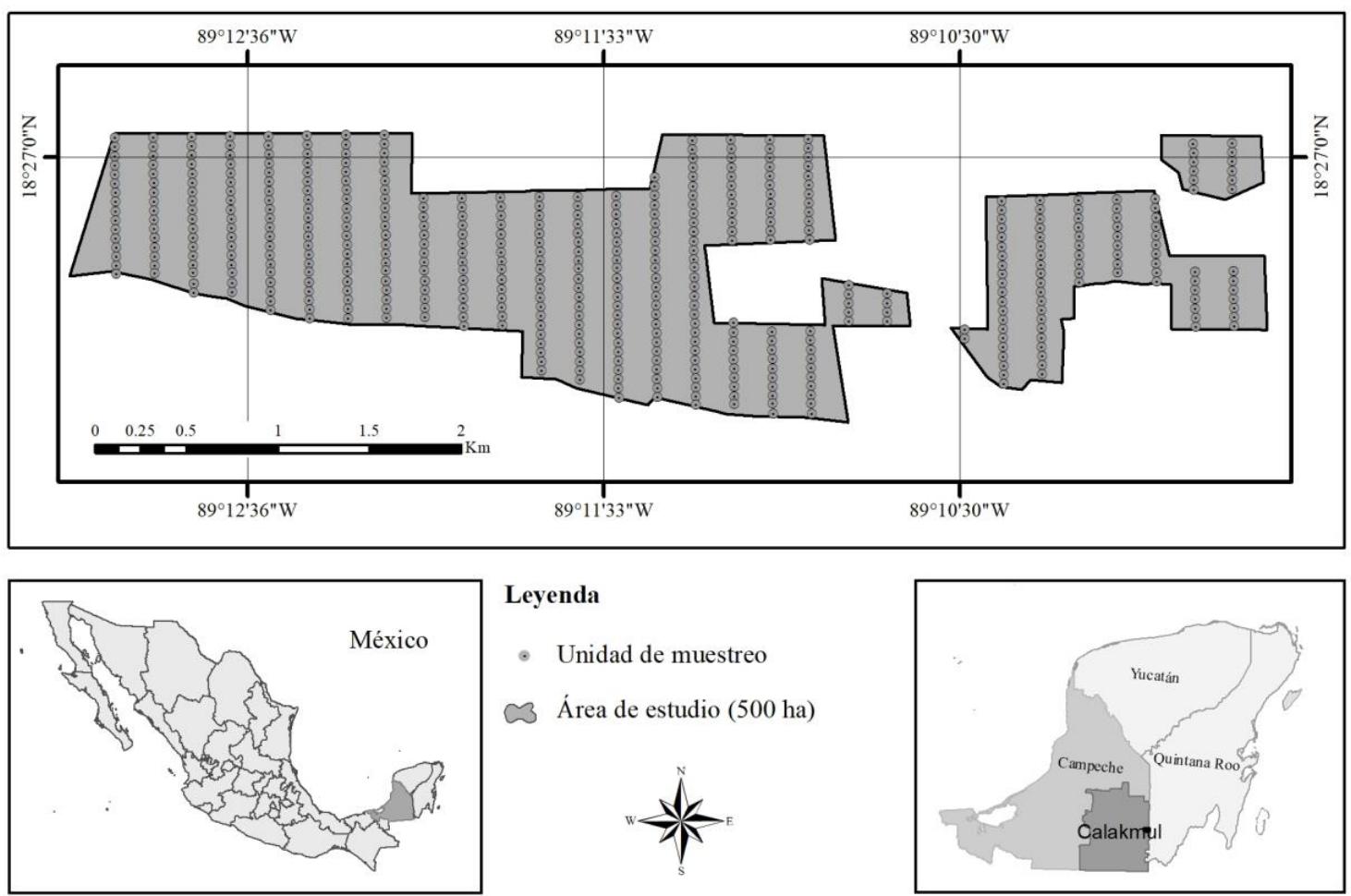

Figura 1. Ubicación geográfica y distribución de los sitios de muestreo en el ejido Gustavo Díaz Ordaz Calakmul, México.

Geographical location and distribution of sampling sites in the ejido Gustavo Díaz Ordaz Calakmul, Mexico.

$$
\operatorname{IVIr}=\left(\mathrm{Dr}+\mathrm{D}_{\mathrm{o}} \mathrm{r}+\mathrm{Fr}\right) / 3
$$

Donde, IVIr = Índice de valor de importancia relativizado, $\mathrm{Dr}=$ Densidad relativa, $\mathrm{D}_{\mathrm{o}} \mathrm{r}$ : Dominancia relativa, $\mathrm{Fr}=$ Frecuencia relativa.

De acuerdo con (Chai et al. 2016), los parámetros que describen al IVIr se detallan en las siguientes fórmulas:

Densidad $\left(\mathrm{D}_{\mathrm{i}}\right)=$ Enúmero de individuos de la especie i/ área muestreada

Densidad relativa $(\mathrm{Dr})=$ densidad de la especie $i\left(\mathrm{D}_{\mathrm{i}}\right) /$ densidad de todas las especies $\left(\sum \mathrm{D}_{\mathrm{i}}\right) \times 100$

Área basal del individuo de la especie $i\left(\mathrm{AB}_{i}\right)=(\pi / 4) \times \mathrm{Dn}^{2}[4]$

$$
\text { Dominancia }\left(\mathrm{Do}_{\mathrm{i}}\right)=\sum \mathrm{AB}_{\mathrm{i}} / \text { área muestreada }
$$

Dominancia relativa $\left(\mathrm{D}_{\mathrm{o}} \mathrm{r}\right)=\left(\right.$ dominancia de la especie $i\left(\mathrm{Do}_{\mathrm{i}}\right) /$

$\sum$ de la dominancia de todas las especies $\left(\sum \mathrm{Do}_{\mathrm{i}}\right) \times 100 \quad$ [6]

Frecuencia $\left(\mathrm{F}_{\mathrm{i}}\right)=$ número de sitios donde se reporta la especie $\mathrm{i} /$ número total de sitios

Frecuencia relativa: frecuencia de la especie $i\left(\mathrm{~F}_{\mathrm{j}}\right) / \sum$ de las frecuencias por especie $\left(\sum \mathrm{F}_{\mathrm{i}}\right)$
Las especies se ordenaron jerárquicamente y en forma descendente de acuerdo con el valor del IVIr, y el análisis del coeficiente de asociación se concentró en las 10 primeras especies, mientras el resto se agruparon en la categoría "otras especies".

Prueba de Chi-cuadrada. De las 10 primeras especies se determinaron 45 pares de combinaciones sin repetición para probar la distribución de la independencia de los datos mediante la prueba de Chi cuadrado corregida $\left(\chi^{2}\right)$ usando la fórmula de corrección de Yates; ecuación 9.

$$
\chi 2=\frac{N\left[(a d-b c)-\left(\frac{N}{2}\right)\right]^{2}}{(a+b)(c+d)(a+c)(b+d)}
$$

Lo anterior a través de una tabla de contingencia $2 \times 2$ (cuadro 1) con un grado de libertad (Chai et al. 2016, Cole 1949, Hurlbert 1969), bajo la hipótesis nula de que la distribución de dos especies es independiente. De hecho, de acuerdo con Chai et al. (2016), esta prueba se considera por si misma un coeficiente de asociación entre pares de especies.

El valor de $\chi^{2}$ para cada uno de los 45 pares de especies se comparó con el valor de $\chi^{2}$ calculado a partir de la $\chi^{2}$ de tablas. Cuando $\chi^{2}<3,841$ no existe asociación entre especies, cuando $3,841 \leq \chi^{2}<6,635$ existe cierta asociación 
Cuadro 1. Tabla de contingencia para evaluar la asociación de especies con base en datos de presencia-ausencia.

Contingency table to evaluate the species association based on presence-absence data.

\begin{tabular}{lllll}
\hline & & \multicolumn{2}{c}{ Especie B } & \multirow{2}{*}{$\sum$} \\
\cline { 3 - 4 } & & Presencia & Ausencia & \\
\hline \multirow{2}{*}{ Especie A } & Presencia & $\mathrm{a}$ & $\mathrm{b}$ & $\mathrm{a}+\mathrm{b}$ \\
& Ausencia & $\mathrm{c}$ & $\mathrm{d}$ & $\mathrm{c}+\mathrm{d}$ \\
& $\sum$ & $\mathrm{a}+\mathrm{c}$ & $\mathrm{b}+\mathrm{d}$ & $\mathrm{N}=\mathrm{a}+\mathrm{b}+\mathrm{c}+\mathrm{d}$ \\
\hline
\end{tabular}

entre especies; mientras si $\chi^{2} \geq 6,635$ denota una asociación significativa entre especies (Whittaker 1975, Chai et al. 2016).

Con el objeto de medir el tipo de interacción entre especies se aplicó el coeficiente de asociación interespecífica $(C A)$ y error estándar $\left(\sigma_{C A}\right)$ propuesto por Cole (1949); en todos los pares donde se rechazó la hipótesis nula (Whittaker 1975, Sanjerehei y Rundel 2020). Las asociaciones se pueden presentar bajo tres condiciones (Rodríguez-Salazar 2000).

Cuando ad $\geq$ bc $\quad \mathrm{CA}=\frac{a d-b c}{(a+b)(b+d)} \quad \sigma_{C A}=\sqrt{ } \frac{(a+b)(c+d)}{N(a+b)(b+d)}$

Cuando $\mathrm{ab}<\mathrm{bc}$ y $\mathrm{a} \leq \mathrm{d}$ CA $=\frac{a d-b c}{(a+b)(a+c)} \sigma_{C A}=\sqrt{ } \frac{(b+d)(c+d)}{N(a+b)(a+c)}$

Cuando ad $<$ bc y a $>$ d CA $=\frac{a d-b c}{(b+d)(c+d)} \sigma_{C A}=\sqrt{ } \frac{(a+b)(a+c)}{N(b+d)(c+d)}$

El índice de CA asume valores desde 1 para asociaciones positivo completo $(b=0, c=0)$ hasta -1 para asociaciones negativo completo $(\mathrm{a}=0, \mathrm{~d}=0)$. Si CA es igual a cero entonces no hay asociación (Chai et al. 2016).

\section{RESULTADOS}

Riqueza y composición. Se registraron 85 especies arbóreas, 32 familias y 36 géneros. Las familias con mayor riqueza fueron Fabaceae (23,5 \%), Sapotaceae (7,1 \%), Moraceae (5,9\%) y Malvaceae $(5,9 \%)$, mientras que las 28 familias restantes acumulan $57,6 \%$ de total de especies. Los géneros más representativos fueron Lonchocarpus $(3,5 \%)$, Pouteria (3,5\%), Nectandra, Caesalpinia y Trichilia (2,3\% cada una, respectivamente). En total se censaron 16.751 árboles, una densidad de 1.398,75 individuos ha ${ }^{-1}$, que aportan una dominancia en área basal de $20,20 \mathrm{~m}^{2} \mathrm{ha}^{-1}(\mathrm{AB})$.
Importancia estructural. La acumulación de los valores del IVIr en 10 especies es de 47,98\%. Las cinco especies con mayor importancia estructural en el ejido Gustavo Díaz Ordaz fueron: B. simaruba (Burseraceae), Croton reflexifolius (Euphorbiaceae), Metopium brownei (Anacardiaceae), Licaria campechiana (Lauraceae) y Lonchocarpus xuul (Fabaceae) (cuadro 2).

Una alta proporción de especies aporta menos de un punto porcentual al IVIr, esto implica una selva de especies raras, muy diversa, pero con poca abundancia por especie; esto es, una baja equitatividad.

Pruebas de asociación de especies. La prueba de $\chi^{2}$ mostró que, entre las poblaciones de árboles dominantes, 17 pares $(37,7 \%)$ tuvieron independencia en la distribución de los datos (figura 2). Las asociaciones fueron positivas, y entre los pares con el mayor valor de $\chi^{2}$ destacan: $B$. simaruba y L. campechiana $\left(\chi^{2}=42,80 ; 0,00<P<0,05\right)$; Croton reflexifolius y Lonchocarpus xuul $\left(\chi^{2}=25,26 ; 0,00<P\right.$ $<0,05)$; y Lonchocarpus castilloi y Lonchocarpus xuul $\left(\chi^{2}\right.$ $=22,5 ; 0,00<P<0,05)$. En general, las asociaciones significativas entre las poblaciones de árboles dominantes fueron significativas $(0,00<P<0,05)$, mientras que el $35 \%$ de los pares presentaron asociaciones débiles entre especies.

Medidas de asociación entre especies. El coeficiente de asociación interespecífica de Cole mostró que, entre las poblaciones de árboles dominantes, 11 pares $(64,7 \%)$ mostraron asociación positiva y seis pares $(35,3 \%)$ tuvieron asociaciones negativas (figura 3). M. brownei se relaciona con seis especies (positiva y negativa). Las asociaciones positivas $(0,15 \leq \mathrm{AC} \leq 1)$ fueron $B$. simaruba con $L$. campechiana; L. xuul con L. castilloi; M. brownei con C. cozumelensis y L. xuul con C. reflexifolius. Las asociaciones negativas $(-1 \leq \mathrm{AC} \leq-0,15)$ fueron $M$. brownei con L. castilloi; $M$. brownei con $S$. mombin y C. cozumelensis con S. mombin.

\section{DISCUSIÓN}

La riqueza arbórea de la selva mediana subperennifolia del ejido Gustavo Díaz Ordaz (85 especies) es mayor a lo consignado por Zamora-Crescencio et al. (2016) y BáezVargas et al. (2017) en Campeche (con 62 y 65 especies, respectivamente), así como por Carreón-Santos y ValdezHernández (2014) en Quintana Roo (54 taxa), con riqueza semejante al ejido Bacalar (86 taxa) (Tadeo-Noble et al. 2019) pero menor a los hallazgos reportados por el mismo autor en el ejido Noh Bec (106 especies) en la parte centro del estado de Quintana Roo. Las familias (Fabaceae, Sapotaceae, Moraceae y Malvaceae) y géneros (Lonchocarpus, Pouteria, Nectandra, Caesalpinia y Trichilia) con la mayor riqueza, así como los taxa arbóreos más abundantes son característicos de la selva mediana subperennifolia de la Península de Yucatán ( Martínez y Leal 2002, ZamoraCrescencio et al. 2016, Gutiérrez-Báez et al. 2017). 
Cuadro 2. Índices de valor de importancia de las especies arbóreas del ejido Gustavo Díaz Ordaz. Importance value indexes of tree species of the ejido Gustavo Díaz Ordaz.

\begin{tabular}{llrrrrrrr}
\hline \multicolumn{1}{c}{ Especie } & \multicolumn{1}{c}{ Familia } & D & Do & F & DR & DoR & Fr & IVIr \\
\hline Bursera simaruba (L.) Sarg. & Burseraceae & 163,84 & 3,88 & 370 & 11,71 & 19,23 & 6,35 & 12,43 \\
Croton reflexifolius Kunth & Euphorbiaceae & 126,93 & 0,74 & 301 & 9,07 & 3,65 & 5,17 & 5,96 \\
Metopium brownei (Jacq.) Urb. & Anacardiaceae & 75,41 & 0,97 & 270 & 5,39 & 4,80 & 4,63 & 4,94 \\
Licaria campechiana (Standl.) Kosterm. & Lauraceae & 88,18 & 0,56 & 317 & 6,30 & 2,76 & 5,44 & 4,84 \\
Lonchocarpus xuul Lundell & Fabaceae & 73,32 & 0,47 & 206 & 5,24 & 2,33 & 3,54 & 3,70 \\
Pouteria campechiana (Kunth) Baehni & Sapotaceae & 50,44 & 0,70 & 231 & 3,61 & 3,48 & 3,96 & 3,68 \\
Spondias mombin L. & Anacardiaceae & 30,40 & 0,99 & 143 & 2,17 & 4,88 & 2,45 & 3,17 \\
Lonchocarpus castilloi Standl. & Fabaceae & 27,39 & 1,03 & 136 & 1,96 & 5,08 & 2,33 & 3,13 \\
Pouteria reticulata (Engl.) Eyma & Sapotaceae & 45,59 & 0,53 & 201 & 3,26 & 2,65 & 3,45 & 3,12 \\
Coccoloba cozumelensis Hemsl. & Polygonaceae & 45,85 & 0,51 & 188 & 3,28 & 2,53 & 3,23 & 3,01 \\
Subtotal (10) & 9 & 727,35 & 10,38 & - & 52 & 51,4 & 40,55 & 47,98 \\
Otras especies (75) & 23 & 671,40 & 9,82 & - & 48 & 48,6 & 59,45 & 52,02 \\
\hline Total & 32 & $1.398,75$ & 20,20 & 479 & 100 & 100 & 100 & 100 \\
\hline
\end{tabular}

D: densidad (árboles ha $\left.{ }^{-1}\right)$; Do: área basal $\left(\mathrm{m}^{2} \mathrm{ha}^{-1}\right)$; F: frecuencia; Dr: densidad relativa (\%); DoR: dominancia relativa (\%); Fr: frecuencia relativa; IVIr: índice de valor de importancia relativo (\%).

D: density $\left(\right.$ tree ha $\left.{ }^{-1}\right), \mathrm{AB}$ : basal area $\left(\mathrm{m}^{2} \mathrm{ha}^{-1}\right), \mathrm{F}$ : frequency, Dr: relative density (\%), DoR: relative dominance (\%), Fr: relative frequency, IVIr: average importance value index $(\%)$.

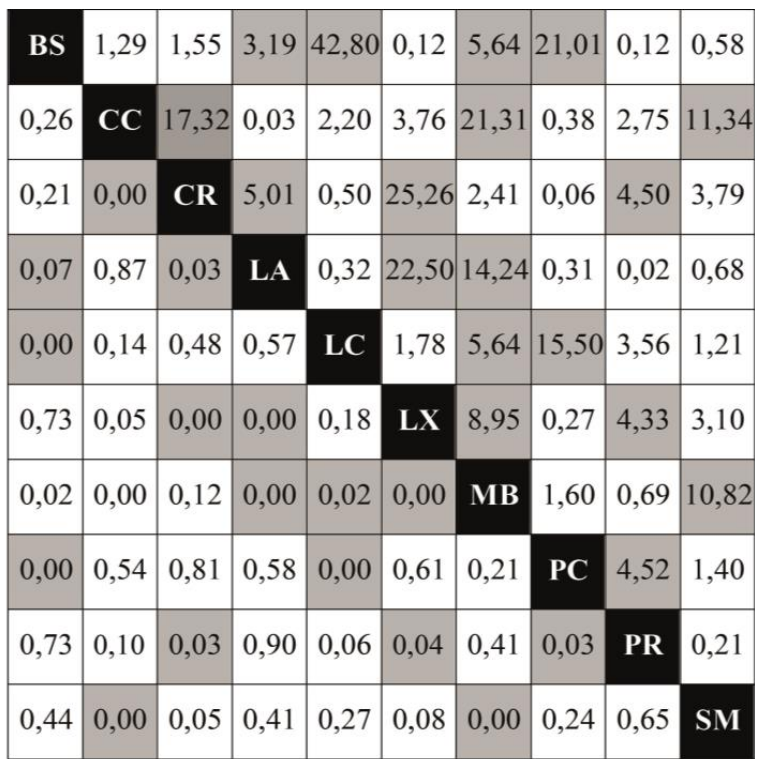

Figura 2. Semimatriz de la prueba de asociación de $\chi^{2}$ entre especies de árboles dominantes en selva mediana subperennifolia de Calakmul, México. Diagonal abajo: P-valor. BS: B. simaruba, CC: C. cozumelensis, CR: C. reflexifolius, LA: L. castilloi, LC: L. campechiana, LX: L. xuul, MB: M. brownei, PC: P. campechiana, PR: P. reticulata, SM: $S$. mombin.

Semi-matrix of the $\chi^{2}$-association test between dominant tree species in the semi-ever green forest of Calakmul, Mexico. Diagonal below: P-value. BS: B. simaruba, CC: C. cozumelensis, CR: C. reflexifolius, LA: L. castilloi, LC: L. campechiana, LX: L. xuul, MB: M. brownei, PC: P. campechiana, PR: P. reticulata, SM: S. mombin.

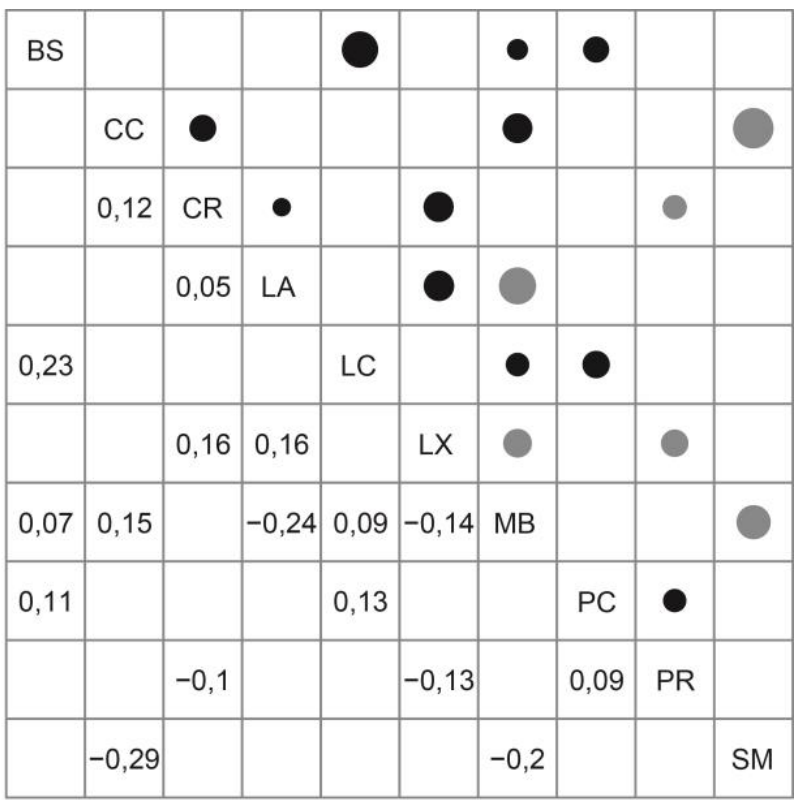

Figura 3. Semigrama de correlación del coeficiente de asociación interespecífica. Diagonal abajo: valor CAI. BS: B. simaruba, CC: C. cozumelensis, CR: C. reflexifolius, LA: L. castilloi, LC: L. campechiana, LX: L. xuul, MB: M. brownei, PC: P. campechiana, PR: P. reticulata, SM: S. mombin.

Semi-gram of correlation of the interspecific association coefficient. Diagonal below: CAI value. BS: B. simaruba, CC: $C$. cozumelensis, CR: C. reflexifolius, LA: L. castilloi, LC: L. campechiana, LX: L. xuul, MB: M. brownei, PC: P. campechiana, PR: P. reticulata, SM: S. mombin. 
De acuerdo con Martínez y Leal (2002), la vegetación del ejido Gustavo Díaz Ordaz representa una asociación de comunidades secundarias de selva, lo que explica la dominancia estructural de aquellas especies con mayor IVI, ya que como lo señalan Tadeo-Noble et al. (2019) y SánchezGutiérrez et al. (2019), este grupo de especies son consideradas pioneras tardías altamente competitivas, derivadas de procesos históricos naturales y de manejo humano que repercuten es su abundancia actual. La especie más dominante fue $B$. simaruba (IVI $=12,43 \%$ ), semejante a lo encontrado por Tadeo-Noble et al. (2019) en Bacalar, Q. Roo $(\mathrm{IVI}=16,6 \%)$, pero mayor a lo que reportan ZamoraCrescencio et al. (2016) en Campeche (IVI = 5,7 \%) y Carreón-Santos y Valdez-Hernández (2014) en la porción centro del estado de Quintana Roo (9,83 \%). Por el contrario, Bursera simaruba, Metopium brownei, Coccoloba spicata, Dendropanax arboreus, Pseudobombax ellipticum y Swietenia macrophylla son especies serales tempranas de vida larga, típicas de selvas perturbadas (Gutiérrez-Granados et al. 2011, Carreón y Valdez 2014, Dzib et al. 2014).

Contrario a la hipótesis planteada, la relación entre especies descrita mediante la $\chi^{2}$ y el coeficiente de asociación interespecífico basado en el IVI presenta una alta proporción de pares con significancia $(P<0,05)$, que coinciden con los estudios realizados en Hongchun Gully, China, donde se registraron asociaciones positivas en mayor proporción que las interrelaciones negativas (Huang et al. 2017). De acuerdo con los criterios de Su et al. (2015) y Chai et al. (2016), las asociaciones interespecíficas positivas y negativas encontradas entre las especies de la selva de Calakmul son débiles.

Los pares de especies que muestran asociaciones positivas se distribuyen dentro de una amplia sobreposición de nichos con recursos semejantes, comportamiento asociado a requerimientos ambientales similares y al desarrollo paralelo de mecanismos de coexistencia, tales como facilitación y modificación de nichos (Chai et al. 2016, Huang et al. 2017, Liu et al. 2017). Por el contrario, las asociaciones negativas denotan que las plantas tienen hábitats y requerimiento de recursos diferentes ( $\mathrm{Su}$ et al. 2015), condición generalmente dada por condiciones específicas de micrositio y capacidad de supervivencia diferencial entre especies (preferencia específica). No obstante, no se descarta densodependencia como una expresión de la intensa competencia interespecífica, debido a que a medida que la frecuencia relativa es menor la presencia de relaciones interespecíficas se disipa en el conjunto de especies que coexisten y su presencia no está ligada a otra especie.

Si bien la función $\mathrm{K}_{12}(t)$ de Ripley también calcula de una forma estadísticamente robusta las asociaciones espaciales interespecíficas entre plantas (Perry et al. 2006, Sanjerehei y Rundel 2020), no estima ni detecta dentro de un conjunto de datos (mayor a dos organismos) a los diferentes pares de especies que mantienen interacciones positivas o negativas. Debido a ello, la novedosa propuesta que presenta este manuscrito, retomada de los planteamientos de Cole (Cole 1949), debe ser considerada como un procedimiento previo e independiente, pero complementario, a la $\mathrm{K}_{12}(t)$ de Ripley, con el objeto de seleccionar con criterio numérico a los taxa que podrían ser sometidos a análisis bivariantes a posteriori, y describir así con mayor precisión la distribución espacial de estos. Cabe mencionar que elegir una ruta de análisis diferente, como es la selección a priori del conjunto de especies, podría disminuir la objetividad e incrementar el sesgo, generando con ello interpretaciones ecológicas erróneas derivadas de interacciones biológicas "forzadas" (Sanjerehei y Rundel 2020). En consecuencia, aplicar la metodología propuesta robustece los análisis de ecología espacial en especies arbóreas tropicales.

\section{CONCLUSIONES}

La información sinecológica obtenida mediante el índice de valor de importancia ayuda a definir el tipo de asociación vegetal del área de estudio como una selva mediana subperennifolia con predominio de B. simarubaC. reflexifolius-M. brownei, que prescribe una asociación interespecífica. El análisis de las relaciones entre las especies a través del coeficiente de asociación interespecífica del Cole contribuye a la explicación de los patrones de distribución espacial, precisando numéricamente la intensidad de la asociación entre especies. Por lo tanto, se propone que esta metodología debería ser empleada como una prueba previa, independiente pero complementaria, a la aplicación de la $\mathrm{K}_{12}(t)$ de Ripley, debido a que proporciona una base estadística para la selección "no artificial" de los taxa bajo análisis.

\section{AGRADECIMIENTOS}

Al despacho consultor Sickingia S. C., por el apoyo en el registro de datos en campo. Al personal de campo del ejido Gustavo Díaz Ordaz, Campeche.

\section{REFERENCIAS}

Báez-Vargas AM, L Esparza-Olguín, E Martínez-Romero, S Ochoa-Gaona, N Ramírez-Marcial, NA González-Valdivia. 2017. Efecto del manejo sobre la diversidad de árboles en vegetación secundaria en la Reserva de la Biosfera de Calakmul, Campeche, México. Revista de Biología Tropical 65(1): 41-53. DOI: https://doi.org/10.15517/rbt.v65i1.20806

Bray JR. 1956. A Study of Mutual Occurrence of Plant Species. Ecology 37(1):21-28. DOI: https://doi.org/10.2307/1929665

Carreón-Santos RJ, JI Valdez-Hernández. 2014. Estructura y diversidad arbórea de vegetación secundaria derivada de una selva mediana subperennifolia en Quintana Roo. Revista Chapingo Serie Ciencias Forestales y del Ambiente 20(1): 119-130. DOI: http://dx.doi.org/10.5154/r.rchscfa.2013.06.023

Chai Z, C Sun, D Wang, W Liu. 2016. Interspecific associations of dominant tree populations in a virgin old-growth oak 
forest in the Qinling Mountains, China. Botanical studies 57(1): 23. DOI: https://doi.org/10.1186/s40529-016-0139-5

Cole LC. 1949. The measurement of Interspecific associaton. Ecology 30(4): 411-424. DOI: https://doi.org/10.2307/1932444

Gutiérrez-Báez C, WJ Folan, L Folan, S Gallegos, P ZamoraCrescencio. 2017. Estructura y composición florística de la selva mediana subcaducifolia de Imi, Campeche, México. Foresta Veracruzana 19(1): 1-8. DOI: http://dx.doi. org/10.18387/polibotanica.43.3

Huang YY, H Han, C Tang, SJ Liu. 2017. Plant community composition and interspecific relationships among dominant species on a post-seismic landslide in Hongchun Gully, China. Journal of Mountain Science 14(10): 1985-1994. DOI: https://doi.org/10.1007/s11629-017-4382-3

Hurlbert SH. 1969. A Coefficient of Interspecific Association. Ecology 50(1): 1-9. DOI: https://doi.org/10.2307/1934657

INEGI (Instituto Nacional de Estadística y Geografía, MX). 2017. Anuario estadístico y geográfico de Campeche 2017. Aguascalientes, México. Instituto Nacional de Estadística y Geografía. 399 p.

Janson S, J Vegelius. 1981. Measures of ecological association. Oecologia 49(3): 371-376. DOI: https://doi.org/10.1007/ BF00347601 https://doi.org/10.1007/BF00347601

Jiménez-Pérez J, E Alanís-Rodríguez, Ó Aguirre-Calderón, M Pando-Moreno, M González-Tagle. 2009. Análisis sobre el efecto del uso del suelo en la diversidad estructural del matorral espinoso tamaulipeco. Madera y Bosques 15(3): 5-20. DOI: https://doi.org/10.21829/myb.2009.1531183

Liu L, X Wang, Q Wen, Q Jia, Q Liu. 2017. Interspecific associations of plant populations in rare earth mining wasteland in southern China. International Biodeterioration \& Biodegradation 118: 82-88. DOI: https://doi.org/10.1016/j. ibiod.2017.01.011

Lot A, F Chiang. 1986. Manual de herbario: administración y manejo de colecciones, técnicas de recolección y preparación de ejemplares botánicos. Distrito Federal, México. Consejo Nacional de la Flora de México. 142 p.

MacKenzie DI, JD Nichols, JA Royle, KH Pollock, L Bailey, JE Hines. 2017. Occupancy estimation and modeling: inferring patterns and dynamics of species occurrence. Londres, Reuino Unido. Elsevier. 648 p.

Martínez E, CG Leal. 2002. La vegetación de Calakmul, Cam- peche, México: clasificación, descripción y distribución. Boletín de la Sociedad Botánica de México 71: 7-32. DOI: https://doi.org/10.17129/botsci.1660

Miranda FP, E Hernández-Xolocotzi. 2014. Los tipos de vegetación de México y su clasificación. Edición conmemorativa 1963-2013/Faustino Miranda y Efraím Hernández-X. México DF, México. FCE, CONABIO, SBM. 214 p.

Perry GL, BP Miller, NJ Enright. 2006. A comparison of methods for the statistical analysis of spatial point patterns in plant ecology. Plant Ecology 187(1): 59-82. DOI: https://doi. org/10.1007/s11258-006-9133-4

Rodríguez-Salazar ME. 2000. Coeficientes de asociación. Madrid, España. Plaza y Valdes. 168 p.

Rzedowski J. 2006. Vegetación de México. México DF, México. Comisión Nacional para el Conocimiento y Uso de la Biodiversidad. 504 p.

Sanjerehei MM, PW Rundel. 2020. A comparison of methods for detecting association between plant species. Ecological Informatics 55(101034): 1-8. DOI: https://doi.org/10.1016/i. ecoinf.2019.101034

Shaukat SS, F Hussain, H Zafar, TA Rao, K Mahmood, A Raza. 2014. Species composition, spatial heterogeneity, interspecific association and diversity of an early successional plant community: A comparison of some species association indices. International Journal of Biology Biotechnology 11(4): 677-691.

Su SJ, JF Liu, ZS He, SQ Zheng, W Hong, DW Xu. 2015. Ecological species groups and interspecific association of dominant tree species in Daiyun Mountain National Nature Reserve. Journal of Mountain Science 12(3): 637-646. DOI: https://doi.org/10.1007/s11629-013-2935-7

Tadeo-Noble AE, JI Valdez-Hernández, L Beltrán-Rodríguez, E García-Moya. 2019. Efecto del aprovechamiento forestal sobre la estructura y diversidad arbórea en selvas tropicales de Quintana Roo, México. Bosque 40(2): 129-140. DOI: http://dx.doi.org/10.4067/S0717-92002019000200129

Whittaker R. 1975. Communities and Ecosystems. New York, EUA. Macmillan Publishing. 162 p.

Zamora-Crescencio P, C Gutiérrez-Báez, P Villegas, MR Domínguez-Carrasco, RC Barrientos-Medina. 2016. Composición y estructura del componente arbóreo de la vegetación secundaria en Campeche, México. Foresta Veracruzana 18(1): 17-24. 
